\title{
Población y vivienda en España: el sistema residencial a la luz del censo de 2001
}

\author{
Juan A. Módenes* \\ Julián López-Colás** \\ *Universitat Autònoma de Barcelona. Departament de Geografia \\ Centre d'Estudis Demogràfics \\ juanantonio.modenes@uab.cat \\ **Universitat Autònoma de Barcelona. Centre d'Estudis Demogràfics \\ jlopez@ced.uab.es
}

Recibido: 10-10-2007

Aceptado: 10-10-2008

\section{Resumen}

El artículo parte del concepto de sistema residencial. Implica la interrelación entre la inserción residencial y la dimensión social y demográfica de los hogares. Se ha estudiado la asociación relativa de distintos rasgos residenciales a la edad de la persona principal de los hogares. El poder explicativo de esta variable se ha controlado mediante la tipología familiar y el tamaño demográfico del municipio donde reside el hogar. Las dimensiones residenciales son la tenencia de la vivienda, el número de plantas del edificio en que se ubica, el año de construcción del edificio y la superficie de la vivienda.

Palabras clave: población, vivienda, España, censos.

Abstract. Population and housting in Spain: the Spanish housing system in light of 2001 census

This paper focuses on the concept of the residential system, which implies the interrelation between households' housing behavior and social and demographic dimensions. The relative association between several housing characteristics and the age of the head of household has been explored. The explanation ability of this variable has been controlled by the structure of the household and the size of the residence locality. The dimensions of housing included in this paper are dwelling tenure, the number of floors of the building, construction year of the building and dwelling's extension.

Key words: population, housing, Spain, census.

\section{Sumario}

La demografía residencial: el encuentro entre población y vivienda

Contexto disciplinario actual

Marco conceptual

Datos y metodología
Resultados

Síntesis y conclusiones

Referencias bibliográficas

Anexo metodológico 


\section{La demografía residencial: el encuentro entre población y vivienda}

La demografía residencial analiza cómo los comportamientos demográficos y las configuraciones familiares interactúan, en el doble sentido, con los contextos residenciales. En el presente artículo introduciremos este enfoque, aprovechando el nuevo potencial que permite el Censo español de Población y Viviendas de 2001.

La historia del estudio de la relación entre población y vivienda ha estado llena de desencuentros (Myers, 1990). En primer lugar, porque ambos polos configuraron disciplinas científicas modernas, excluyentes entre sí al principio, la demografía y la urbanística. Y porque se desarrollaron procesos estadísticos paralelos de generación de información demográfica y residencial, sin una interconexión eficiente antes de la era informática.

En las últimas décadas, estos déficits se han ido superando. El desarrollo de la multidisciplinariedad también ha afectado a la población y a la vivien$\mathrm{da}$, incentivada por la necesidad de estudios aplicados, como por ejemplo las previsiones de demanda residencial. Además, la revolución informática ha favorecido la disponibilidad de fuentes estadísticas, como por ejemplo los actuales censos de población y vivienda, que combinan ambas dimensiones.

Se hará a continuación referencia a cuatro puntos que ilustran este proceso de reencuentro: el concepto de demografía residencial, el concepto de estrategia residencial, el de sistema residencial y los nuevos desarrollos en España.

\section{Contexto disciplinario actual}

El término «demografía residencial» (housing demography) ha sido utilizado en el ámbito anglosajón para referirse a esta temática interdisciplinaria, que reúne enfoques y metodologías de ambas disciplinas para proyectar los comportamientos residenciales de la población (Myers, 1990; Gober, 1992; sin olvidar Rossi, 1955). El enfoque basado en el concepto de estrategia residencial se desarrolló en Francia durante la década de 1980 y se ha plasmado en una serie de obras colectivas (Bonvalet y Merlin, 1988; Bonvalet y Fribourg, 1990; Lelièvre y Levy-Vroelant, 1992; Grafmeyer y Dansereau, 1998). En ellas, se estudia la racionalidad del comportamiento residencial de los hogares en relación con sus redes sociales próximas y el contexto residencial inmediato. El hogar se considera un agente autónomo que juega entre las restricciones y las oportunidades del contexto (en parte, residencial), las motivaciones individuales, el desarrollo familiar y las normas sociales.

Otra aproximación, tanto o más interesante, que ha priorizado la escuela francesa es el estudio del contexto residencial como condicionante de las estrategias de inserción urbana de la población. Esta escuela ha aportado una visión dinámica y sistémica del funcionamiento del polo residencial en conexión con el desarrollo sociodemográfico de la población. Los conceptos de sistema residencial, espacio residencial y tipo residencial (Dureau, 1999 y 2002; Lévy, 1998, 2002 y 2003) ejemplifican este interés. 
España no ha sido ajena a estos desarrollos. Los temas son comunes a los tratados internacionalmente: cadenas de vacantes, elementos contextuales y familiares en la comprensión de las estrategias residenciales y de localización, acceso a la propiedad en las estrategias familiares y estimaciones de demanda, entre otros. Desde la sociología, Jesús Leal (Leal y Cortés, 1995; Leal, 1997, 2004; Allen, Barlow, Leal et al., 2004) y Cortés $(1995,2005)$ han realizado importantes aportaciones, contemporáneas a las ya mencionadas. También han de destacarse las aportaciones de economistas (Pareja, 1999; Pareja y San Martín, 2003, Taltavull, 2000) o geógrafos (Pujadas y García Coll, 2005; Vinuesa, 2003, 2007).

Desde la demografía española, se están realizando interesantes contribuciones. El Grupo de Investigación sobre el Envejecimiento del CSIC analiza las pautas de inserción residencial de las personas de edad avanzada (Fernández-Mayoralas et al., 2002 y 2004; Puga y Abellán, 2002; Puga, 2004). En el Centre d'Estudis Demogràfics, se ha analizado la base sociodemográfica de la experiencia residencial, integrando progresivamente el contexto residencial en la investigación (Cabré y Módenes, 2004; Domingo y Bayona, 2002 y 2005; Domingo, Bayona y López Gay, 2004; López Colás y Módenes, 2004, 2005; Módenes, 2001; Módenes y López Colás, 2004, 2007a, 2007b, 2007c).

\section{Marco conceptual}

Esta contribución se organiza alrededor de los conceptos de sistema y tipo residencial. El concepto de sistema residencial ha sido adoptado por muchos investigadores que se mueven en este cruce entre lo familiar y lo urbano. Françoise Dureau (1999) lo utiliza a una escala inferior a la que utilizaremos nosotros. Define sistema residencial como la interrelación entre los lugares accesibles a las estrategias residenciales y las prácticas residenciales y familiares de los hogares urbanos. La residencia se convierte en una dimensión inherente al desarrollo familiar, que toma forma como una sucesión temporal o combinación simultánea de lugares y viviendas. Dureau insiste más en las localizaciones residenciales, lo que ella denomina espacio residencial, que en las características de las viviendas. En esta segunda opción, encontramos a Jean Pierre Lévy (1998), que utiliza el concepto de tipo residencial como catalizador del polo de la vivienda en el conjunto del sistema. El tipo residencial reúne en un mismo instrumento las características de las viviendas, la posición sociodemográfica de los hogares ocupantes y la interpretación sociodemográfica de las viviendas que esta relación implica. Las tipologías residenciales no serían, pues, un concepto meramente arquitectónico, sino que tendrían una dimensión social y demográfica. El concepto de espacio residencial, en su visión, nos remite a un espacio abstracto bidimensional formado por las tipologías de vivienda y las fases temporales del curso de vida familiar. Las distintas combinaciones reales de ambas dimensiones forman la serie de tipos residenciales (normalmente a escala urbana). 


\section{Datos y metodología}

Estudiaremos las propensiones relativas de los hogares a vivir en determinado tipo de viviendas según la principal dimensión demográfica, la edad de la persona principal ${ }^{1}$. Las dimensiones residenciales que se han escogido en este artículo son la tenencia de la vivienda ${ }^{2}$, el número de plantas del edificio en que se ubica, el año de construcción del edificio y la superficie de la vivienda ${ }^{3}$. Normalmente, se suele insistir más en la distribución del tipo de tenencia para caracterizar la inserción residencial de una sociedad (Kurz y Blossfeld, 2004). Como veremos, en el caso de España, el predominio de la propiedad de la vivienda principal en todo el espectro sociodemográfico obliga a matizar más el análisis de esta variable para detectar los elementos de diversidad que subyacen, así como a introducir otros rasgos residenciales que pueden evidenciar la heterogeneidad de la inserción residencial.

La tenencia no es una característica intrínseca de la vivienda, pero define la relación de ocupación, legal, económica e, incluso, emocional, entre el hogar y su casa. Ofrece información sobre las cargas financieras que debe soportar el hogar para residir en esa vivienda, la previsión de finalización en un futuro y el grado de estabilidad de la residencia.

El número de plantas del edificio nos aproxima al tipo de contexto urbanístico, en que se inserta la residencia del hogar. Por lo general, existe una relación estrecha entre la densidad constructiva de un hogar y el valor medio de los hogares próximos, del vecindario. Otros estudios nos han confirmado la potencia de esta variable, por ejemplo, para explicar la posesión de viviendas secundarias, como práctica compensatoria de la elevada densidad de la vivienda principal (Módenes y López Colás, 2007a).

El año de construcción del edificio es un rasgo dinámico y estático a la vez. Este dato puede interpretarse, al mismo tiempo, como edad o como cohorte de construcción de la vivienda. Como edad, es un elemento dinámico según el cual las características y la calidad de la vivienda cambiarían con el paso del tiempo, suponiendo que cuanto más reciente es una vivienda, en mejores condiciones está. Como cohorte constructiva, es una variable estática, según la cual una vivienda acarrearía durante toda su vida una serie de elementos estables, propios del momento en que se construyeron (estilo constructivo, disposición de espacios, tipos de materiales), independientemente de la edad alcanzada. Estos rasgos pueden determinar la valoración, económica y social,

1. Se ha preferido la edad de la mujer para construir esta variable en los casos en que forma parte del núcleo principal, porque es más indicativa de la etapa del ciclo de vida familiar del hogar. Los grupos de edad contemplados son: <30, 30-44, 45-59, 60-74 y 75 o más años de edad.

2. Las categorías son propiedad heredada o donada, propiedad totalmente pagada, propiedad con pagos pendientes, alquiler, cesión y otras formas.

3. Estas variables se han organizado en seis categorías, en orden jerárquico: número de plantas (1, 2, 3, 4, 5-7, 8 y más), año de construcción (<1960, 1961-1970, 1971-1980, 1981$1990,1991-1996,1997-2001)$ y superficie $\left(<60,61-75,76-90,91-120,121-150\right.$ y $\left.>150 \mathrm{~m}^{2}\right)$. 
de esas viviendas en el momento presente. Por eso, es interesante referirse más al año de construcción y no tanto a la antigüedad de la vivienda ${ }^{4}$. Asimismo, hay que tener presente que cuando un edificio ha sido reformado sustancialmente, el Instituto Nacional de Estadística (INE) imputa la fecha de la reforma como año de construcción.

Por último, la superficie de la vivienda nos aproxima mejor a su calidad y a su comodidad. También es la que mejor expresa el nivel socioeconómico de sus ocupantes. Podemos interpretar que el tamaño va en relación directa con la calidad y la estabilidad de la residencia. Por supuesto, en viviendas pequeñas es posible desarrollar actividades familiares, pero la vinculación del hogar con esa vivienda tenderá a ser menos estable, a no ser que se limite el número de miembros del hogar. Un tamaño suficiente, al que cabría añadir otros elementos de calidad, garantiza una mayor duración temporal del hogar en la vivienda y se asegura una mayor estabilidad residencial.

Toda la información estadística ha sido extraída del Censo de Población de $2001^{5}$. Se ha realizado un tratamiento descriptivo de la misma, aunque introduciendo algunos elementos propios del análisis demográfico clásico como la estandarización de indicadores. Esta metodología clásica se emparienta con métodos estadísticos más sofisticados en que la base común es el control del efecto de una tercera o más variables en la relación entre dos variables en estudio (Menacho, 2002). No se han interconectado las diferentes características residenciales entre sí, aunque sí se han controlado las interferencias estructurales que sobre la edad de la persona principal tienen la tipología familiar y el tamaño del municipio de residencia.

El objetivo de la estandarización es encontrar indicadores sintéticos y resumidos que puedan mostrar las verdaderas diferencias en un fenómeno determinado, cuando se comparan dos o más poblaciones distintas o una misma población en períodos de tiempo sucesivos. En nuestro caso, el fenómeno es múltiple y se identifica con los distintos rasgos residenciales enunciados. Nuestro objetivo es comparar el comportamiento de cada categoría de edad con el conjunto de hogares, aislando el máximo de interferencias de los otros rasgos sociodemográficos, ya que la edad no es independiente del tipo de hogar ni de la localización geográfica. Por ejemplo, compararemos el comportamiento de los hogares encabezados por personas de menos de treinta años con el conjunto de hogares, considerando el efecto neto de tener esta edad, independientemente de la influencia de las otras variables sociodemográficas; también compararemos la mayor importancia en estas edades de estructuras no nucleares y de su mayor presencia relativa en el medio urbano. Si no realizáramos este con-

4. En Estados Unidos se utiliza el concepto de vintage, que se traduce como 'cosecha' o 'añada', como los vinos (por ejemplo, en Myers, 1990). El concepto capta esta idea que las «añadas» de viviendas son buenas o malas no porque sean antiguas o recientes, sino por ciertas características propias del momento de construcción.

5. Hemos explotado, con el paquete estadístico SPSS, un fichero de microdatos con la muestra del 5\% del total de los hogares españoles. 
trol, podríamos atribuir a un efecto de edad ciertos comportamientos que tienen más que ver, eventualmente, con el comportamiento de los hogares no nucleares o urbanos ${ }^{6}$.

En los gráficos, se ha utilizado una trama clara para destacar aquellas situaciones que juzgamos más desfavorables para los hogares (tener cargas financieras, vivir en extrema densidad urbanística, habitar una vivienda muy antigua o disponer de pocos metros cuadrados en la residencia principal). Por el contrario, la trama más intensa nos conduce hacia las frecuencias asociadas a las características más favorables (no tener ningún tipo de carga y gozar de seguridad jurídica absoluta respecto de la vivienda, evitar un exceso de densidad ${ }^{7}$, habitar en un edificio reciente o en una vivienda de gran superficie).

Sistemáticamente, se ofrece una pareja de gráficos. El primero presenta la distribución relativa estandarizada de cada categoría de edad según las categorías de la variable residencial en cuestión. El segundo introduce el análisis de las diferencias estandarizadas de los comportamientos específicos respecto del comportamiento agregado. Para esto último, hemos medido sistemáticamente la diferencia absoluta entre las proporciones específicas de los hogares respecto a la misma proporción del conjunto de hogares. Se ha pretendido conducir visualmente al lector hacia la identificación de la heterogeneidad en la experiencia residencial de los hogares españoles.

En este análisis gráfico de la selectividad en las preferencias residenciales, se han establecido algunos criterios visuales para mejorar la interpretación. Consideramos que vale la pena hablar de cierta selectividad positiva o negativa cuando la diferencia entre el indicador específico y el agregado supera los tres puntos porcentuales. La selectividad es intensa cuando la diferencia supera los diez puntos porcentuales. Si los casos de selectividad intensa abundan, es obligado hablar de la presencia de fuertes elementos de heterogeneidad en el comportamiento residencial.

Por último, se ha construido un cuadro sinóptico que resume los casos de heterogeneidad demográfica existentes en las cuatro dimensiones analizadas del sistema residencial español a la luz del censo de 2001.

6. Hemos supuesto que la variable analizada (edad de la persona principal), al igual que las variables sociodemográficas de control (estructura familiar, tamaño del municipio), son independientes entre sí, es decir, que determinados tipos de hogar no se concentran excesivamente en edades concretas, o que en los tramos demográficos municipales habita una parecida composición por edad y tipología familiar. Como es obvio, este supuesto analítico no es totalmente real, y las interferencias entre las variables demográficas pueden ser intensas. Estas interferencias también pueden estudiarse desde el punto de vista del análisis del sistema residencial. Véase el anexo para una pequeña introducción a este tipo de aproximación.

7. Nótese que estamos hablando de los elementos favorables a la inserción residencial de los hogares. Creemos que, a igualdad del resto de elementos (servicios, comercio, amenidades), un hogar preferirá el mínimo de congestión urbanística. No prejuzgamos cuáles son las opciones residenciales más apropiadas en el contexto general de la ordenación territorial del uso residencial del suelo. 
Tabla 1. Categoría residencial más frecuente de los hogares según edad de la persona principal, por variable residencial (España, 2001)

\begin{tabular}{|c|c|c|c|c|}
\hline \multirow{2}{*}{$\begin{array}{l}\text { Variables demográficas } \\
\begin{array}{l}\text { Edad de la persona } \\
\text { principal }\end{array}\end{array}$} & \multicolumn{4}{|c|}{ Variables residenciales } \\
\hline & Tenencia* & $\begin{array}{l}\text { Plantas } \\
\text { del edificio }\end{array}$ & Año construcción & Superficie $\left(\mathrm{m}^{2}\right)$ \\
\hline$<30$ & Propiedad PP & $5-7$ & $<1960$ & $76-90$ \\
\hline $30-44$ & Propiedad PP & $5-7$ & $<1960$ & $76-90$ \\
\hline $45-59$ & Propiedad TP & $5-7$ & $1971-1980$ & $76-90$ \\
\hline $60-74$ & Propiedad TP & $5-7$ & $<1960$ & $76-90$ \\
\hline $75+$ & Propiedad TP & $5-7$ & $<1960$ & $76-90$ \\
\hline
\end{tabular}

Fuente: elaboración propia a partir del Censo de Población de 2001.

*PP: con pagos pendientes (hipotecas...), TP: totalmente pagada.

\section{Resultados}

Una primera exploración somera refleja una imagen muy homogénea del sistema residencial (tabla 1). Todos los hogares, independientemente de la edad de la persona principal, suelen vivir en contextos de alta densidad urbanística (5-7 plantas) y en viviendas de tamaño intermedio $\left(76-90 \mathrm{~m}^{2}\right)$. En cuatro de las cinco categorías de edad, la categoría de año de construcción más frecuente es la de antes de 1960. Algo más diversas son las propensiones a la tenencia: los hogares de menos de cuarenta y cinco años son mayoritariamente de propietarios con pagos pendientes, mientras que los de más edad son de propietarios sin ningún tipo de pago pendiente por la vivienda.

Sin duda, es un rasgo propio del sistema español esta primera capa de uniformidad, en la tenencia, en los tipos de hábitat o en el resto de rasgos residenciales. Sin embargo, el objetivo de este artículo es indagar los elementos de heterogeneidad presentes en el sistema residencial. Para ello, se representará gráficamente y se comentará la distribución de los hogares, detallados por cada grupo de edad de la persona principal, según cada una de las cuatro variables residenciales, así como las diferencias respecto al comportamiento agregado.

\section{La tenencia de la vivienda}

El censo de 2001 confirma el dominio de la propiedad de la vivienda principal: el $82,22 \%$ de los hogares españoles vive en régimen de propiedad frente al $11,30 \%$, que lo hace en régimen de alquiler. Analizar la heterogeneidad del sistema residencial español a partir de la oposición entre alquiler y propiedad es insuficiente. Interesa también combinar dos ejes: cargas financieras y estabilidad de la relación con la vivienda. El censo de 2001 nos acerca mejor a la existencia de cargas financieras (aunque no a su magnitud ${ }^{8}$ ) que a la estabilidad

8. No sabemos la cuantía del alquiler, ni de los pagos por hipoteca, ni tampoco si la cesión representa una carga financiera, por pequeña que sea. 
A

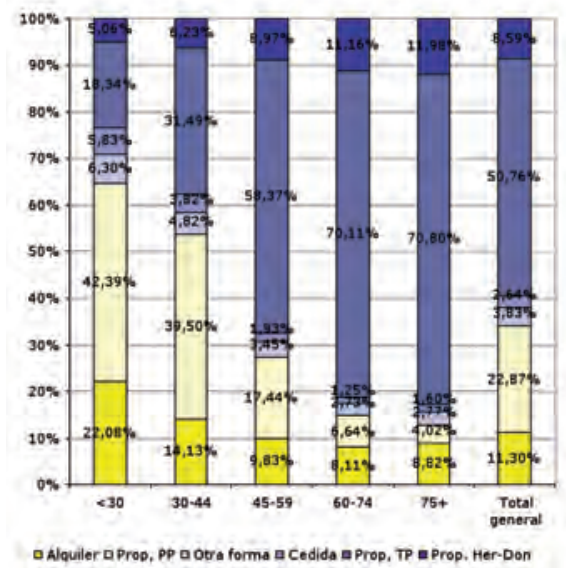

B

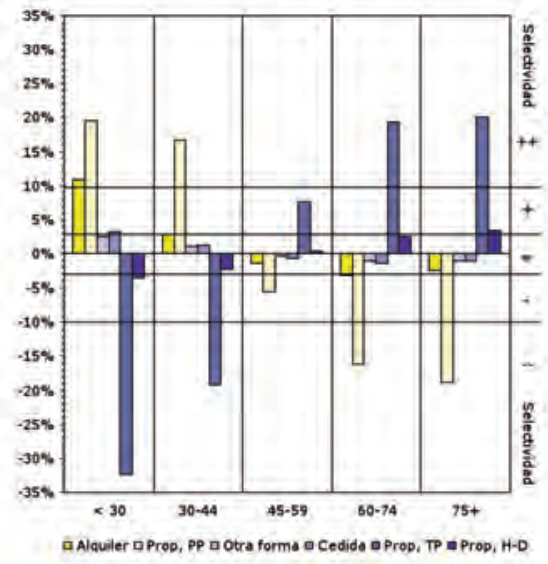

Figura 1. Tenencia de la vivienda por edad de la persona principal del hogar. A) Distribución relativa estandarizada de la tenencia por edad. B) Diferencias relativas estandarizadas de cada respecto del conjunto de hogares (España, 2001). Fuente: INE, Censo de Población de 2001.

del vínculo9. Por ello, se han reordenado jerárquicamente las categorías en función del grado de compromiso financiero regular. La categoría que vincula más sería el alquiler; la que menos, la propiedad por herencia o donación. En el primer caso, los pagos son permanentes (mientras no se cambie de régimen de tenencia), en el segundo, teóricamente nunca se ha realizado ningún pago (excepto, eventualmente, el impuesto de sucesiones). Un hogar tiene mejor inserción residencial cuanto mayor sea su grado de libertad actual respecto de las cargas económicas. No se pueden conocer las eventuales dificultades del pasado.

Bajo este prisma, la situación media de los hogares con respecto a la tenencia de su vivienda es relativamente positiva. Sólo una tercera parte de los hogares españoles efectúa pagos regulares, de los cuales, dos tercios viven en propiedad con pagos pendientes (normalmente, pagando una hipoteca) y el resto está en alquiler. La mitad de los hogares está disfrutando de una vivienda que acabó de pagar antes de la fecha del censo. Por último, el 8,6\% de los hogares habita una vivienda que ha heredado ${ }^{10}$.

9. Puede suponerse una estabilidad total en los casos de propiedad por herencia y propiedad sin pagos pendientes. La cesión puede ser temporal. El alquiler puede ser en régimen de contrato indefinido. No sabemos si el propietario con pagos pendientes tiene problemas de morosidad que afecte a su relación con la vivienda.

10. Esta última cifra debe de ser inferior a la de hogares que han recibido alguna vez una vivienda o parte de una en herencia. Algunos de éstos se habrán desprendido de ella (por venta o donación) y no viven allí. Para otros hogares, será una vivienda secundaria o la mantienen vacía. 
La tenencia es muy sensible a la edad de la persona principal (figura 1) y, por lo tanto, a la duración del hogar ${ }^{11}$. Los compromisos financieros disminuyen con la duración del hogar, puesto que se parte mayoritariamente de una situación de propietario con pagos pendientes. El alquiler es más frecuente en los hogares jóvenes, aunque es una opción minoritaria. Los hogares encabezados por una persona de edad avanzada viven más en herencia, lo que puede deberse a un efecto de edad (es a esos años cuando se reciben las herencias) o de generación (los hogares más jóvenes preferirían movilizar el valor económico de una vivienda heredada y no ocuparla).

\section{El número de plantas del edificio de la residencia principal}

Casi un $45 \%$ de los hogares vive en edificios de cinco o más plantas de altura; un $18 \%$, en edificios de tres o cuatro plantas; un $21 \%$, en edificios de dos plantas, y un $16 \%$, en edificios de una planta. La suma de las dos últimas categorías se acerca al total de edificios unifamiliares, fijado en un 30\%. Estas proporciones son muy propias del sistema residencial español y encuentran pocos casos semejantes entre los países europeos próximos, sólo en la Europa mediterránea.

Los hogares españoles, independientemente de la edad y de su etapa de desarrollo, viven en un contexto residencial parecido (figura 2). Predomina en todas las edades un medio urbano bastante denso, ya que siempre más del $40 \%$ de los hogares, sean jóvenes, maduros o viejos, viven en edificios de cinco plantas o más. El contexto urbanístico dominante en el momento de la formación de los hogares explicaría una cierta selectividad de los hogares en que la persona principal es de edad avanzada en viviendas de baja altura y de los hogares encabezados por personas de mediana edad en los entornos más densos.

\section{Año de construcción de la vivienda}

El parque español es relativamente reciente. En 2001, sólo el 27,6\% del parque tenía más de cuarenta años de antigüedad. Las viviendas con menos de veinte años (construidas entre 1981 y 2001) representaban casi el 30\%, cuando en censos anteriores no sobrepasaba el 7\% del total. Este elevado porcentaje de viviendas nuevas coloca a España en una posición intermedia en el contexto europeo ${ }^{12}$. Desde finales de la década de 1990, España es el tercer país en

11. Dada la baja proporción relativa de disolución por divorcio y reconstitución de nuevos hogares, puede suponerse que la edad de la persona principal es una aproximación indirecta al tiempo que lleva constituido el hogar.

12. Sólo Portugal, Irlanda y Finlandia tendrían una proporción mayor de viviendas recientes (National Agency for Enterprise and Housing, 2004: 31), aunque las cifras de esta publicación se refieren al total del parque de viviendas, no sólo a las viviendas principales. Por otro lado, España también es el país europeo con un porcentaje mayor de vivienda muy antigua. 
A

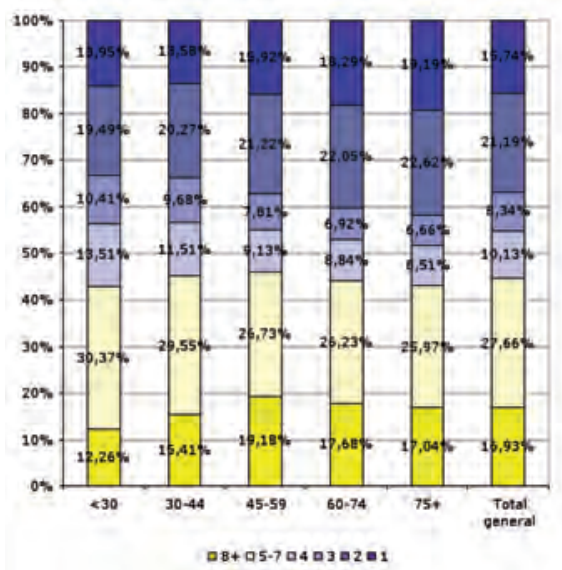

B

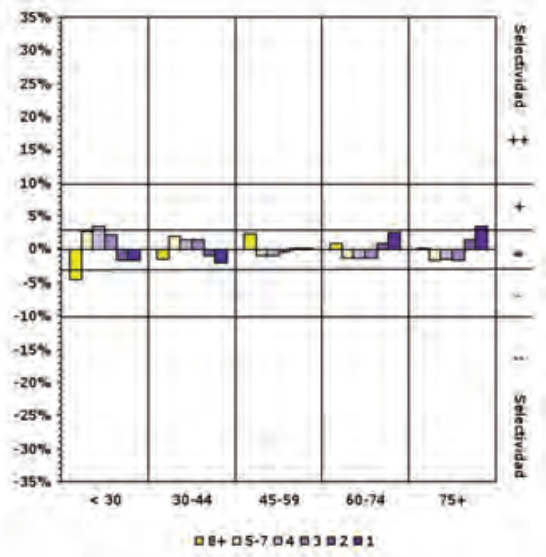

Figura 2. Número de plantas del edificio por edad de la persona principal del hogar. A) Distribución relativa estandarizada del número de plantas por edad. B) Diferencias relativas estandarizadas de cada edad respecto del conjunto de hogares (España, 2001). Fuente: INE, Censo de Población de 2001.

construcción de viviendas por cada mil habitantes ${ }^{13}$, el primero entre los grandes países europeos.

La edad del hogar, considerada a partir de la edad de la persona principal, y la edad de la vivienda correlacionan de manera directa. A más edad del hogar, se habita en una vivienda más antigua (figura 3). Esta relación es tanto más cierta cuanto menos móviles sean los hogares (lo que se ha cumplido hasta bien recientemente) y cuanto más acceso a viviendas nuevas tengan los hogares jóvenes (lo que es más difícil). El sentido de la tendencia afloja en los hogares más jóvenes, con un elevado acceso a viviendas de segunda mano.

Si al año de construcción le atribuimos valores de calidad, debemos fijarnos en la cohorte de la vivienda y no solamente en la edad. Si consideramos que la vivienda construida en los años sesenta presenta una baja calidad dentro del conjunto, los hogares de 60-74 años, los que más habitan estas viviendas, son los más perjudicados del sistema.

\section{La superficie de la vivienda}

Más del 30\% de los hogares españoles dispone de menos de $75 \mathrm{~m}^{2}$ en su vivien$\mathrm{da}^{14}$. En un $14 \%$ de los casos la vivienda tiene menos de $60 \mathrm{~m}^{2}$. Más de la

13. Detrás de Irlanda y Portugal (National Agency for Enterprise and Housing, 2004: 45).

14. No hemos estudiado la ratio entre la superficie y el número de miembros del hogar, que complementaría los resultados. 
A

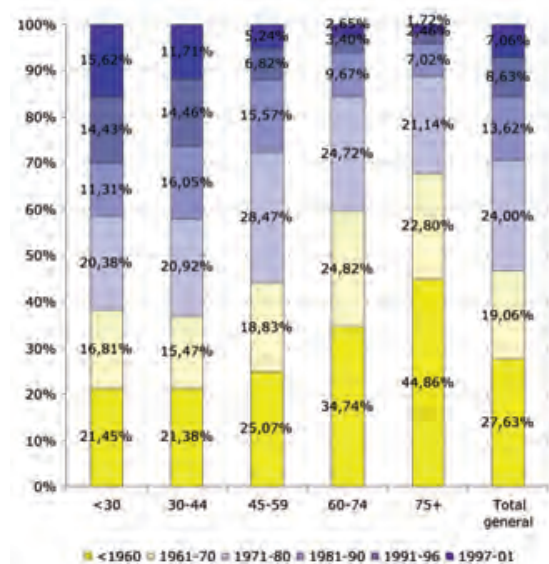

B

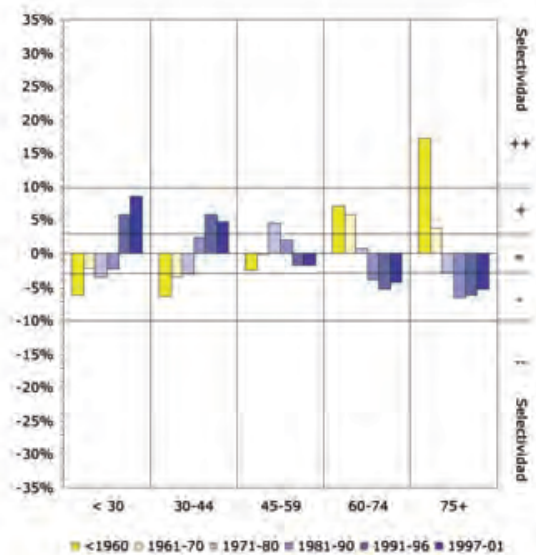

Figura 3. Año de construcción del edificio por edad de la persona principal del hogar. A) Distribución relativa estandarizada del año de construcción por edad. B) Diferencias relativas estandarizadas de cada edad especto del conjunto de hogares (España, 2001). Fuente: INE, Censo de Población de 2001.
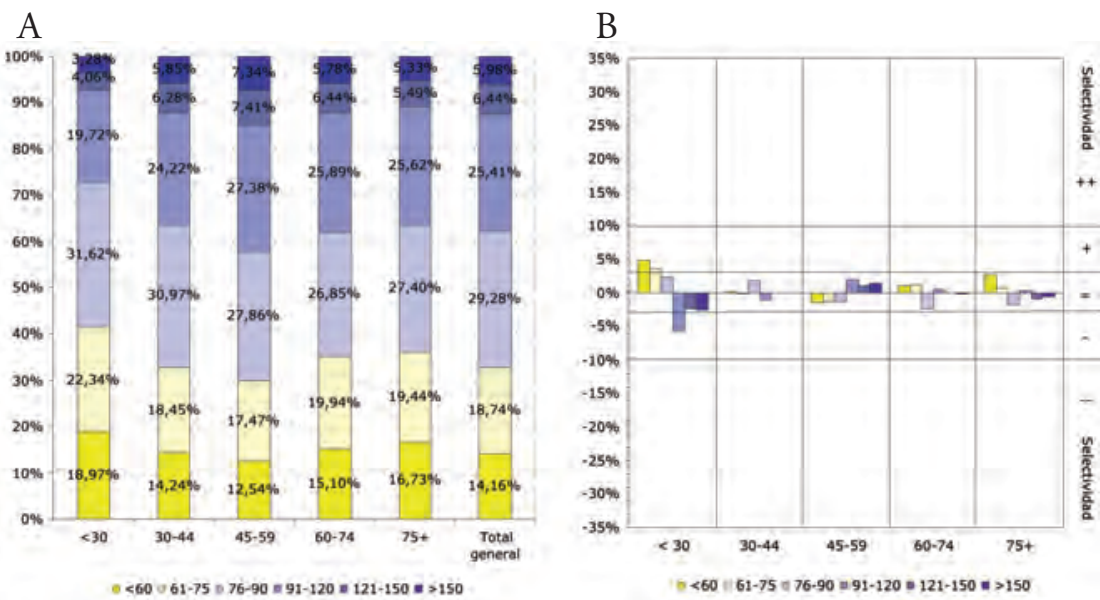

Figura 4. Superficie de la vivienda por edad de la persona princiapl del hogar. A) Distribución relativa estandarizada de la superficie por edad. B) Diferencias relativas estandarizadas de cada edad respecto del conjunto de hogares (España, 2001). Fuente INE, Censo de Población de 2001. 
mitad de los hogares se distribuyen casi equitativamente en los tamaños intermedios, 76-90 y 91-120 $\mathrm{m}^{2}$. Es muy escasa, algo más del 12\%, la presencia de viviendas principales de más de $120 \mathrm{~m}^{2}$. En el contexto europeo, España está situada en una posición intermedia. La superficie media de una vivienda familiar es de $90 \mathrm{~m}^{2}$, valor bastante normal entre el resto de países. Sin embargo, hay que tener en cuenta que, en el conjunto de hogares españoles, hay un menor peso de hogares unipersonales y hogares de jóvenes recién emancipados, por lo que seguramente la comparación internacional sobre la base de estructuras familiares estandarizadas sería desfavorable para España.

En España, la relación entre superficie de la vivienda y edad de la persona principal del hogar no es muy importante (figura 4). Existe un cierto efecto de edad en los primeros grupos, de manera que, cuanto mayor es la persona principal, más se eleva paulatinamente la proporción de viviendas grandes. El grupo de edad que disfruta de las viviendas más grandes es el de 45-59 años. Los grupos posteriores vuelven a tener viviendas más pequeñas, más bien por un efecto generacional: los hogares más viejos han habitado desde tiempo atrás en esas viviendas.

\section{Síntesis y conclusiones}

Se ha cumplido el objetivo inicial, que era la identificación de los eventuales ejes de heterogeneidad y diversidad existentes en el modelo residencial español, caracterizado por la propiedad, la alta densidad, la estabilidad residencial y la presencia de viviendas pequeñas en todo tipo de hogares. Reunamos los dos niveles de análisis del sistema residencial a que hemos aludido en este estudio introductorio. Por un lado, los rasgos comunes. Por otro, aquellos elementos diferenciales que hemos ido identificando a lo largo del presente artículo y que permiten captar las dimensiones de heterogeneidad presentes en el sistema residencial español.

La tabla 2 recoge los casos de selectividad específica en que la distancia entre los valores de la categoría de edad y del conjunto es suficientemente amplia. Como síntesis, se han identificado, en cada categoría de hogar, las dos categorías residenciales (en cada una de las cuatro variables de vivienda) que más se alejan del valor del conjunto, una por exceso y la otra por defecto ${ }^{15}$.

Hay una fuente principal de heterogeneidad en el sistema residencial español. Se trata de la evolución de la tenencia según la edad de la persona principal. En el polo totalmente opuesto, comprobamos que no hay inte-

15. Así, por ejemplo, en el caso del número de plantas del edificio y en los hogares cuya persona principal no ha cumplido los treinta años de edad, hemos detectado que el porcentaje en cuatro plantas es el más alejado del valor del conjunto por el lado positivo y el valor de la categoría $8+$ es el más alejado por el lado negativo. Es decir, estos hogares jóvenes tienden a vivir bastante más que el conjunto en edificios de cuatro plantas y tienden a residir bastante menos que el conjunto en edificios de 8+ plantas de altura, aunque su frecuencia más alta está en los edificios de cinco a siete plantas, como el resto de categorías de edad. 
Tabla 2. Situación residencial más frecuente y selectividades positivas y negativas significativas según característica demográfica. Tenencia y número de plantas (España, 2001)

\begin{tabular}{|c|c|c|c|c|c|c|}
\hline \multirow{2}{*}{$\begin{array}{l}\text { Edad de } \\
\text { la persona } \\
\text { principal }\end{array}$} & \multicolumn{3}{|c|}{ Tenencias } & \multicolumn{3}{|c|}{ Plantas } \\
\hline & $1^{\text {a }}$ frecuen. & Sel.(+) & Sel.(-) & $1^{\text {a }}$ frecuen. & Sel.(+) & Sel.(-) \\
\hline$<30$ & Prop. PP & Prop. PP & Prop. TP & $5-7$ & 4 & $8+$ \\
\hline $30-44$ & Prop. PP & Prop. PP & Prop. TP & $5-7$ & & \\
\hline $45-59$ & Prop. TP & Prop. TP & Prop. PP & $5-7$ & & \\
\hline $60-74$ & Prop. TP & Prop. TP & Prop. PP & $5-7$ & & \\
\hline $75+$ & Prop. TP & Prop. TP & Prop. PP & $5-7$ & 1 & \\
\hline \multirow{2}{*}{$\begin{array}{l}\text { Edad de } \\
\text { la persona } \\
\text { principal }\end{array}$} & \multicolumn{3}{|c|}{ Año construcción } & \multicolumn{3}{|c|}{ Superficie } \\
\hline & $1^{\text {a }}$ frecuen. & Sel.(+) & Sel.(-) & $1^{\text {a }}$ frecuen. & Sel.(+) & Sel.(-) \\
\hline$<30$ & $<1960$ & $1997-2001$ & $<1960$ & $76-90$ & $<60$ & $91-120$ \\
\hline $30-44$ & $<1960$ & 1991-1996 & $<1960$ & $76-90$ & & \\
\hline $45-59$ & $1971-1980$ & 1971-1980 & & $76-90$ & & \\
\hline $60-74$ & $<1960$ & $<1960$ & 1991-1996 & $76-90$ & & \\
\hline $75+$ & $<1960$ & $<1960$ & $1981-1990$ & $76-90$ & & \\
\hline
\end{tabular}

Fuente: elaboración propia a partir del censo de 2001.

Nota: Trama gris: diferencia superior a $>10$ puntos porcentuales respecto del comportamiento de conjunto. En blanco, diferencia inferior a 3 puntos porcentuales.

racción significativa entre la edad y el número de plantas, y la edad y la superficie.

En cuanto a la tenencia, a más edad de la persona principal del hogar, más se reduce substancialmente el nivel de cargas en el pago de la vivienda. En la segunda mitad de la vida de los hogares, disminuye substancialmente la necesidad de efectuar pagos económicos, lo que alivia la reducción de ingresos vinculada con la jubilación. Este comentario es especialmente interesante en el caso de los hogares de edad avanzada, los cuales, como apunta la literatura internacional especializada (Doling y Horsewood, 2003), disfrutan de una especie de renta especial, tanto monetaria como de estabilidad en la inserción residencial, por ocupar viviendas de su propiedad. Hemos identificado la edad de cuarenta y cinco años como el umbral de paso de la primera etapa con más compromisos financieros y menos estabilidad en la vinculación con la vivienda a la segunda etapa de más seguridad y menos cargas. Sin embargo, la evolución actual de las duraciones medias de las hipotecas (en torno a treinta años o más) hace pensar que se trata de un umbral móvil que puede ir avanzando en el futuro próximo hacia edades más elevadas. El alquiler no ofrece una vía residencial a los hogares jóvenes, sino específicamente a la minoría de hogares no estándar, los cuales no pueden acceder fácilmente a la propiedad. 
Todas las edades ocupan frecuentemente viviendas antiguas, lo que demuestra la importancia del mercado de segunda mano para el acceso a la propiedad, especialmente de los jóvenes. En un segundo nivel, el análisis de selectividad muestra que a más edad de la persona principal del hogar suele ser mayor la antigüedad de la vivienda, lo que denota una cierta estabilidad de los hogares que prolonga la duración temporal media de la residencia. Por otro lado, la edad es poco sensible a aspectos formales o físicos de la vivienda, como el número de plantas (o densidad del entorno) y la superficie de la vivienda. Si acaso, los hogares más jóvenes son capaces de dejar de lado algo más los espacios más densos, pero deben entrar más frecuentemente en viviendas pequeñas.

Para finalizar, algunas palabras sobre las condiciones de acceso al sistema residencial español. El ingreso de los hogares jóvenes en el sistema residencial español se realiza en condiciones muy particulares, compartidas por el conjunto de países que definen un modelo residencial sur europeo (Allen et al., 2004). Los hogares jóvenes, menores de treinta años, son mayoritariamente propietarios, tienen contraídas hipotecas u otros compromisos financieros, y habitan viviendas relativamente nuevas y no excesivamente pequeñas. Muchos jóvenes ocupan viviendas que son aptas para el desarrollo ulterior de las necesidades de sus hogares. Las cargas financieras de los hogares jóvenes se proyectan no en el alquiler, sino en la propiedad, pero requieren a corto plazo una mayor capacidad de ahorro que en otros países, una mayor involucración de la red familiar y un mayor compromiso con los otros miembros del hogar, lo que puede explicar en parte la relativamente alta preferencia por el matrimonio (Módenes y López Colás, 2007c). Sin embargo, el hecho que los hogares jóvenes sean mayoritariamente propietarios permite conseguir antes un elevado grado de estabilidad residencial, al igual que favorece la inserción local del hogar y de sus miembros, aunque introduzca un período de exigente estrés financiero.

Para ulteriores aportaciones, se pretende avanzar en la caracterización del sistema español, mediante la construcción teórica del conjunto ordenado de tipos residenciales, como intersección de la temporalidad y la estructura del hogar y su inserción residencial, además de la introducción de nuevas dimensiones sociodemográficas.

\section{Referencias bibliográficas}

Allen, J.; Barlow, J.; Leal, J. et al. (2004). Housing \& Welfare in Southern Europe. Oxford: Blackwell Publishing (Real Estate Issues). 228 p.

Bonvalet, C. y Fribourg, A.M. (ed.) (1990). Stratégies résidentielles. París: INEDPlan Construction et Architecture, MELTM. (Congrès et Colloques; 2)

Bonvalet, C. y Merin, P. (ed.) (1988). Transformation de la famille et habitat. París: L'Harmattan. (Villes et Enterprises)

Cabré, A. y Módenes, J.A. (2004). «Home-ownership and social inequality in Spain». En: KURZ, K. y BLOSSFELD, H.P. (ed.). Social inequality and home-ownership in a comparative perspective. Standford University, 233-254. 
CORTÉS, L. (1995). La cuestión residencial. Bases para una sociología del habitar. Madrid: Fundamentos. (Ciencias-Serie Sociología; 205)

- (2005). "La crisis de la vivienda». Monográfico «Vivienda y alojamiento». Documentación Social, 138, 81-100.

DOLING, J.F. y HORSEWOOD, N.J. (2003). «Home ownership and early retirement: European experience in the 1990s». Journal of Housing and the Built Environment, $18,289-308$.

DOMINGO, A. y BAYONA, J. (2002). «Habitatge i assentament de la població de nacionalitat estrangera als barris de Barcelona, 1991-2001». Qüestions d'Habitatge, 8, 1-26.

- (2005). «Immigració i territori: Concentració i segregació al municipi de Barcelona, 1991-2002». Barcelona Societat, 13, 114-128.

DOMINGO, A.; BAYONA, J. y LÓPEZ GAY, A. (2004). «Impacto demoespacial de la internacionalización de los flujos migratorios en la ciudad de Barcelona». Migraciones, $16,157-198$.

Dureau, F. (1999). "Les mobilités à géométrie variable des habitants de Bogota». Espace, Populations, Sociétés, 1999, 329-344.

- (2002). «Les systèmes résidentiels: concepts et applications». En: LÉVY, J.-P. y DurEau, F. L'accès à la ville. Les mobilités spatiales en questions. París: L'Harmattan, 355-382.

Fernández-Mayoralas, G.; Rojo, F. y Pozo, E. (2002). «El entorno residencial de los mayores en Madrid». Estudios Geográficos, 63 (248/249), 541-575.

FERnÁndeZ-Mayoralas, G.; Rojo, F. y Rojo, J.M. (2004). "Components of the residential environment and sociodemographic characteristics of the elderly». Journal of Housing for the Elderly, 18 (1), 25-49.

Grafmeyer, Y. y DANSEREAU, F. (ed.) (1998). Trajectoires familiales et espaces de vie en milieu urbain. Lyon: Presses Universitaires de Lyon.

Gober, P. (1992). "Urban housing demography». Progress in Human Geography, 16 (2), 171-189.

KURZ, K. y BlOSSFELD, H.P. (ed.) (2004). Social inequality and home-ownership in a comparative perspective. Standford University.

LEAL, J. (1997). "Emancipación y vivienda». En: VerGÉs, R. (ed.). La edad de emancipación de los jóvenes. Barcelona: Centre de Cultura Contemporània de Barcelona, 113-124. (Urbanitats, 1)

- (2004). «Dinámica de la población y desarrollo del parque de viviendas en España». En: LEAL, J. (coord.) (2004). Informe sobre la situación demográfica en España, 2004. Madrid: Fundación Fernando Abril Martorell, 325-352.

LEAL, J. y CORTÉS, L. (1995). La dimensión de la ciudad. Madrid: Siglo XXI, CIS. (Monografías; 145)

Lelièvre, E. y Levy-Vroelant, C. (ed.) (1992). La ville en mouvement: Habitat et habitants. París: L'Harmattan. (Villes et Enterprises)

LÉVY, J-P. (1998). «Habitat et habitants: position et mobilité dans l'espace résidentiel». En: GrafmeYer, Y. y DANSEREAU, F. (ed.). Trajectoires familiales et espaces de vie en milieu urbain. Lyon: Presses Universitaires de Lyon, 153-180.

- (2002). «The population make-up of residential areas: Structure and changes (Douai, France)». Journal of Housing and the Built Environment, 17, 293-319.

- (2003). "Population patterns and household trajectories in the residential mileu: the example of the Seine-Saint-Denis». Population, 58 (3), 323-357.

LÓPEZ COLÁS, J. y MÓdENES, J.A. (2004). «Vivienda secundaria y residencia múltiple en España: una aproximación sociodemográfica». Geo Crítica: Scripta Nova. 
Revista Electrónica de Geografía y Ciencias Sociales. Barcelona: Universidad de Barcelona, 1 de diciembre, vol. vIII, núm. 178.

- (2005). «Segona residència i multiresidència a Catalunya: una aproximació sociodemogràfica». Documents d'Anàlisi Geogràfica, 46, 41-62.

MENACHO, T. (2002). Los tipos de estandarización en demografía: Aplicación al estudio de las diferencias regionales de la actividad y el desempleo en España, 1990-2000. Memoria de investigación de tercer ciclo. Departament de Geografia de la UAB, mimeo.

Módenes, J.A. (2001). «Relacions sòcio-territorials i mobilitat residencial a l’àrea de Barcelona». Revista Catalana de Sociologia, 14.

MÓdENES, J.A. y LÓPEZ COLÁS, J. (2004). «Movilidad residencial, trabajo y vivienda en Europa». Scripta Nova: Revista Electrónica de Geografía y Ciencias Sociales. Universidad de Barcelona, 15 de febrero, vol. VIII, núm. 159.

- (2007a). «Second homes and compact cities in Spain: Two elements of the same system?». Tijdschrift voor Economische en Sociale Geografie, 98 (3), 325-335.

- (2007b). «La résidence secondaire en Espagne: Profils socio-démographiques et territoriaux». Population. Versión francesa: 62 (1), 161-177. Versión inglesa: Population-E, 62 (1).

- (2007c). «Constitución familiar y régimen de tenencia de la vivienda: España en el contexto europeo». En CABrÉ, A. y Miret, P. (eds.). La constitución familiar en España. Bilbao: Fundación BBVA, 199-243.

MYERS, D. (1990). «Introduction: The emerging concept of housing demography». En MYERS, D. (ed.). Housing demography. Linking demographic structure and housing markets. Madison: University of Wisconsin, 3-31. (Social Demography)

National Agency for EnTerprise And Housing (2004). Housing Statistics in the European Union 2003. Copenhague: National Agency for Enterprise and Housing.

PAREJA, M. (1999). Les cadenes de vacants i l'efecte de filtratge: una aplicació a la Vila Olimpica del Poblenou. Universitat Autònoma de Barcelona. Tesis doctoral.

Pareja, M. y SAn Martín, I. (2003). «El sistema de vivienda en España». Situación Inmobiliaria. BBVA, octubre, 23-32.

PugA, D. (2004). Estrategias residenciales de las personas de edad. Movilidad y curso de vida. Barcelona: Fundación la Caixa, 297.

PugA, D. y ABEllán, A. (2002). «Movimientos migratorios y curso de vida: causas y patrones espaciales». Estudios Geográficos, LXIII, 248/249, 733-759.

PUJADAS, I. y GARCía COLL, A. (2005). «De la ciudad compacta a la ciudad dispersa: Movilidad residencial en la Región Metropolitana de Barcelona 1982-2000». XXV Congreso Internacional de Población, sesión 120 («La evolución de patrones de distribución espacial de la población en países altamente urbanizados»). IUSSP. Tours (Francia).

Rossi, P.H. (1955). Why families move: A study in the social psychology of urban residential mobility. Nueva York: Free Press.

Taltavull, P. (coord.) (2000). Vivienda y familia. Argentaria. Visor. Programa de Economía Familiar. (Colección Economía Española; 13)

VINUESA, J. (2003). «Población y demanda de vivienda: una relación a reconsiderar». En: La dinámica geodemográfica protagonista del territorio Actas del VIII Congreso de la Población Española, 283-290.

- (2007). Estimación y caracterización de la demanda de vivienda en la Comunidad de Madrid por ámbitos geográficos (2006-2016). Madrid: Fundación Asprima. 


\section{Anexo metodológico}

Como se ha dicho en la nota 6 , el sistema residencial puede ser completado con la referencia a las interferencias estructurales entre las variables demográficas. Sus efectos sobre los indicadores han sido corregidos en el proceso de la estandarización de los indicadores específicos estudiados en este artículo. Sin embargo, estos efectos y las correcciones estadísticas efectuadas tienen interés. En nuestro caso, que analiza la edad de la persona principal, la estandarización ha corregido las interferencias producidas, tanto por la tipología familiar de esos hogares, como por su localización territorial en municipios de distinto tamaño.

La tabla recoge aquellos casos en que la estandarización ha corregido substancialmente, a la baja o al alza (más de un punto porcentual), los indicadores relativos. Se identifican los casos más importantes de interferencia entre la variable demográfica analizada, la edad de la persona principal y alguna de las otras dos de control (tipo de hogar y tamaño del municipio). Así, por ejemplo, puede observarse que los hogares en que la persona principal tiene entre 30 y 44 años, los propietarios tienden a tener menos pagos pendientes, no sólo a causa de su edad, sino también porque forman con más frecuencia tipos de

Tabla. Mayores correcciones efectuadas mediante el proceso de estandarización e identificación de la estructura que interfiere (España, 2001)

\begin{tabular}{|c|c|c|c|c|}
\hline \multirow{2}{*}{$\begin{array}{c}\text { Edad de la } \\
\text { persona } \\
\text { principal }\end{array}$} & \multicolumn{2}{|c|}{ Tenencia } & \multicolumn{2}{|c|}{ Plantas } \\
\hline & $\begin{array}{c}\text { Corrección } \\
\text { negativa }\end{array}$ & $\begin{array}{c}\text { Corrección } \\
\text { positiva }\end{array}$ & $\begin{array}{c}\text { Corrección } \\
\text { negativa }\end{array}$ & $\begin{array}{c}\text { Corrección } \\
\text { positiva }\end{array}$ \\
\hline \multicolumn{5}{|l|}{$<30$} \\
\hline $30-44$ & Prop. TP & Alquiler & & \\
\hline $45-59$ & Prop. TP & & $8+$ & 1 \\
\hline \multicolumn{5}{|l|}{$60-74$} \\
\hline $75+$ & Prop. H-D & Prop. TP & 1 & $5-7$ \\
\hline \multirow{2}{*}{$\begin{array}{c}\text { Edad de la } \\
\text { persona } \\
\text { principal }\end{array}$} & \multicolumn{2}{|c|}{ Año construcción } & \multicolumn{2}{|c|}{ Superficie } \\
\hline & $\begin{array}{c}\text { Corrección } \\
\text { negativa }\end{array}$ & $\begin{array}{c}\text { Corrección } \\
\text { positiva }\end{array}$ & $\begin{array}{c}\text { Corrección } \\
\text { negativa }\end{array}$ & $\begin{array}{c}\text { Corrección } \\
\text { positiva }\end{array}$ \\
\hline \multicolumn{5}{|l|}{$<30$} \\
\hline $30-44$ & 1981-1990 & $<1960$ & $91-120$ & $<60$ \\
\hline $45-59$ & $1971-1980$ & & & $<60$ \\
\hline $60-74$ & $<1960$ & & $<60$ & \\
\hline $75+$ & $<1960$ & $1971-1980$ & $<60$ & $91-120$ \\
\hline
\end{tabular}

Código de tramas: 
hogar que también son propensos a esa forma de tenencia (el indicador estandarizado de 30 a 44 años ha controlado y ha obviado este efecto conjunto de la estructura de hogar y su valor se ha corregido suavemente a la baja). Análogamente, los hogares en que la persona principal tiene entre 30 y 44 años tienden poco al alquiler, en parte porque forman tipos de hogar poco propensos al alquiler como son las parejas. La estandarización ha controlado este efecto de la estructura de hogar y ha elevado suavemente la proporción de inquilinos en este grupo de edad. Pero es interesante retener la existencia de esas interferencias, por ejemplo, entre edad y tipos de hogar, o edad y tamaño del municipio, que influyen en las elecciones residenciales de las viviendas reales.

Cuando pocos o ningún efecto se encuentra en el cruce entre la variable edad y una variable residencial, se debe a que la distribución de la variable edad es independiente de las otras dos variables demográficas de control. O, más probablemente, porque la sensibilidad entre edad de la persona principal y variable residencial es tan clara que cualquier interferencia eventual de otra de las variables demográficas de control pierde toda importancia.

Por lo que respecta a la tenencia, se observa que los hogares de 30 a 59 años se inclinan más a la propiedad totalmente pagada, en parte también porque, normalmente, forman hogares propensos a esta forma de tenencia (parejas). Esta relación entre tener edades centrales y ser pareja (propia del sistema familiar español) contribuye a explicar la elevada propensión de estos hogares por la propiedad, por viviendas de edad aproximadamente igual al hogar y por superficies relativamente grandes. Igualmente, la interferencia entre hogares de edades avanzadas y solitarios contribuye a su preferencia por viviendas pequeñas. La estructura de tamaño del municipio interfiere más en los hogares viejos, porque éstos están más presentes en el medio rural que el conjunto del país. Incentiva la presencia en estas edades de la herencia o la donación, de viviendas de una altura y de edificios antiguos entre estos hogares. La localización preferente de los hogares de 45 a 59 años en las grandes ciudades contribuye a su mayor presencia en los estratos más densos del parque residencial. 